Dicle University Journal of Engineering (DUJE)

\title{
Bir Mikro Şebekenin Yük Frekans Kontrolü için Tamsayı Derece Yaklaşımlı Kesir Dereceli PID Kontrolörün Optimizasyonu
}

\section{Optimization of Fractional Order Controller with Integer Order Approximation for Load Frequency Control of a Microgrid}

\author{
Burak Yıldırım ${ }^{*}$ \\ ${ }^{1}$ Bingöl Üniversitesi, Teknik Bilimler MYO, Bingöl, byildirim@bingol.edu.tr
}

\begin{tabular}{|c|c|}
\hline MAKALE BİLGİLERİ & ÖZET \\
\hline Makale geçmişi: & \multirow{6}{*}{$\begin{array}{l}\text { Modern güç sistemlerinde yük değişiklikleri ve arızalar sonrası üretim-yük dengesini koruma yeteneğini sağlamak } \\
\text { önemli bir problemdir. Bir yük frekans kontrol (LFC) mekanizması bu gereksinime bir çözüm sağlar. Mikro } \\
\text { şebekelerde üretilen güç miktarı sürekli olarak değişir ve aynı zamanda birçok belirsizliğe sahiptir, bunun nedeni } \\
\text { mikro şebekelerin genellikle elektrik enerjisi üretmek için yenilenebilir enerji kaynakları (RES) kullanmasıdır. } \\
\text { Mikro şebeke sistemlerindeki bu değişiklikler ve belirsizlikler nedeniyle, geleneksel kontrolörler uzun vadede iyi } \\
\text { bir performans sağlamada yetersiz kalmıştır. Bu çalışmada, mikro şebekede, LFC karşılaşılan zorluklarla başa } \\
\text { çıkmak için tamsayı derece yaklaşımlı kesir dereceli PID kontrolör (IOA FOPID) önerilmiştir. En uygun kontrolör } \\
\text { parametrelerinin belirlenmesi için lig şampiyonası algoritması (LCA), karınca koloni optimizasyonu (ACO) ve } \\
\text { optikten esinlenen optimizasyon (OIO) algoritmaları kullanılmıştır. Aynı zamanda, IOA FOPID kontrolörünün } \\
\text { kazançlarının en uygun değerlerinin elde edilmesi için çok amaçlı bir maliyet fonksiyonu kullanılmıştır. Üç farkl1 } \\
\text { optimizasyon algoritması ile elde edilen en uygun kontrolör parametre değerleri için mikro şebeke sistemin zaman } \\
\text { domeni analizleri yapılmıs ve algoritmaların başarıları karssıllastırılmıștır. }\end{array}$} \\
\hline Geliș: 1 Temmuz 2020 & \\
\hline Düzeltme: 1 Eylül 2020 & \\
\hline Kabul: 1 Eylül 2020 & \\
\hline Anahtar kelimeler: & \\
\hline $\begin{array}{l}\text { Mikro şebeke, Tamsayı derece } \\
\text { yaklaşımlı kesir dereceli PID } \\
\text { kontrolör, Çok amaçlı maliyet } \\
\text { fonksiyonu. }\end{array}$ & \\
\hline
\end{tabular}

Doi: $10.24012 /$ dumf. 860298

\begin{tabular}{|c|c|}
\hline ARTICLE INFO & ABSTRACT \\
\hline & \multirow{6}{*}{$\begin{array}{l}\text { In modern power systems, ensuring the ability to maintain production-load balance after load changes and failures } \\
\text { is an important problem. A load frequency control (LFC) mechanism provides a solution to this requirement. The } \\
\text { amount of power produced in microgrids is constantly changing and also has many uncertainties, because } \\
\text { microgrids often use renewable energy sources (RES) to generate electrical energy. Due to these changes and } \\
\text { uncertainties in microgrid systems, traditional controllers have become inadequate to provide good performance } \\
\text { in the long run. In this study, in order to deal with the difficulties encountered in LFC in microgrid, integer order } \\
\text { approximation fractional order PID controller (IOA FOPID) is proposed. League championship algorithm (LCA), } \\
\text { ant colony optimization (ACO) and optics-inspired optimization (OIO) algorithms have used to determine the } \\
\text { most appropriate controller parameters. At the same time, a multi-purpose cost function has used to obtain the } \\
\text { most appropriate values of the gains of the IOA FOPID controller. For the most appropriate controller parameter } \\
\text { values obtained with three different optimization algorithms, the time domain analyzes of the microgrid system } \\
\text { have made and the success of the algorithms has compared. }\end{array}$} \\
\hline Received: 1 July 2020 & \\
\hline Revised: 1 September 2020 & \\
\hline Accepted: 1 September 2020 & \\
\hline Keywords: & \\
\hline $\begin{array}{l}\text { Microgrid, Integer order } \\
\text { approximation fractional order } \\
\text { PID controller, Multi objective } \\
\text { cost function }\end{array}$ & \\
\hline
\end{tabular}

* Sorumlu yazar / Correspondence

Burak YILDIRIM

$\triangle$ byildirim@bingol.edu.tr 


\section{Giriș}

Elektrik için artan talep ve belirsizliklere sahip karmaşık elektrik güç sistemleri geleneksel olmayan güç kaynaklarının güç sistemlerine dahil edilmesine yol açmıştır. Yenilenebilir enerji kaynakları (RES) modern güç sistemlerinde bir alternatif üretim birimi olarak sıklıkla kullanılmaktadır. RES'lerin artan penetrasyon oranları güç sistemlerine birçok avantajlar sunar. Bununla birlikte, bu kaynakların geleneksel üretim birimleriyle çalışıp çalışmadığı veya tek başlarına veya diğer sistemlerle kullanıldıklarında kararlı çalışıp çalışmadığı gibi birçok yeni sorunlarda ortaya çıkmıştır. RES'lerin hem şebekede hem de şebekenin dışında çalışması için uygun kontrol tasarımı, RES'lerin güç sisteminin gerilim ve frekansının düzenlenmesine katkısı ve RES'lerin korunması, vb., gibi sorunlar RES'lerin neden olduğu teknik sorunlardan bazılarıdır [1]. $\mathrm{Bu}$ kaynakların kullanılması birçok standardı da beraberinde getirmiştir. Yukarıda bahsedilen avantaj ve sorunların bir sonucu olarak 1998 yılında ilk kez CERTS tarafından mikro şebeke fikri ortaya çıkmıştır. CERTS tarafından sunulan mikro şebeke, hem güç hem de 1sı sağlayan bir tek sistem olarak çalışan yük ve mikro kaynakların bir birleşiminden oluşmaktadır. Mikro șebeke sistemler mikro kaynakların fazla sayısından dolayı ve tüm bu kaynakların birleşiminden oluşan tek bir sistem sağlamak için elektronik tabanlı olmalıdır [2]-[4].

Mikro şebekelerin ortaya çıkması geleneksel güç sistemlerinin karşılaştığı ekonomik ve çevresel sorunların artmasına dayanır. RES'leri kullanan mikro şebekeler küresel 1sınma ve çevresel problemleri azaltma yetenekleri sayesinde güç endüstrisinde hızlı bir şekilde yaygınlaşmıştır [4], [5].

Elektrik şebekelerinde elektrik arzı ve talebi dengelenmelidir. $\mathrm{Bu}$ ikili durum arasındaki boşluktaki artış, elektrik güç sisteminde gerilim ve frekans sapmalarına neden olur. Frekans ve gerilimdeki bu sapmalar, bu tür sistemlerin kararlılığını ciddi şekilde tehdit eder ve bu bile büyük elektrik kesintilerine neden olabilir [6]. Mikro şebeke için ana problemlerden biri frekans kontrolüdür. Mikro şebeke sisteminin istikrarlı bir şekilde çalışabilmesi ve sistemin frekansını nominal değerde tutabilmesi için üretim ve talep arasında gerçek zamanlı bir eşleşme sağlanması şarttır. Bir yük frekansı kontrol (LFC) mekanizması bu gereksinime bir çözüm sağlar [7]. Mikro şebekeler adalı [8] veya şebekeye bağlı [9] mod olarak çalıştırılır. Adalı modda çalışan mikro şebeke ana şebekeden destek almadığından, LFC bu moddaki en önemli çalışma görevlerinden biri olacaktır [10]. Ref. [11]'de, fotovoltaik (PV), akü enerji depolama sistemi (BESS) ve katı oksit yakıt hücresine dayalı mikro şebekenin frekans / gerilim kontrolü için bulanık mantık kontrolörü önerilmiştir. Ref. [10]'da, adalanmış bir mikro şebekenin LFC'sinin sekonder ve üçüncül kontrolü arasındaki koordinasyonu gerçekleştirmek için optimal aktif güç kontrolü önerilmektedir. Ref. [12]'de, mikro şebekelerin LFC'si için hedefe göre ayarlanmış model öngörülü kontrol önerilmiştir.

Son zamanlarda, kesirli dereceli sistemler büyük ilgi görmüştür. Bunun nedeni kesirli diferansiyel denklemlerin gerçek dünya sistemlerini tamsay1 diferansiyel denklemlerden daha iyi tanımlamalarıdır. Kesirli dereceden hesaplama fizik ve kimya sistematiğinin yanı sıra elektrik devresi, kontrol ve kaos teorileri ve sistem tasarımında da önemli bir rol oynamaktadır [13]. Ancak, kesirli dereceli bir transfer fonksiyonunun analitik ters laplace dönüşümü olmadığından, onun kesin bir zaman cevabını tahmin etmek mümkün değildir. Bu nedenle aynı davranışı sergileyecek olan tamsayı dereceli transfer fonksiyonu, belirli bir bant aralığ 1 boyunca kesirli dereceli transfer fonksiyonu yerine kullanılabilir. $\mathrm{Bu}$ amaçla Oustaloup yöntemi, Sürekli Kesirli Genişleme (CFE) yöntemi, Matsuda yöntemi, Chareff yöntemi, Carlson yöntemi, en küçük kare yöntemleri gibi tamsayı derece yaklaşım yöntemleri kullanılır [14]-[21].

Bu çalışmada, bir IOA FOPID kontroller mikro şebekenin LFC için kullanılmıştır. Bu yönüyle çalışma literatürde bir ilk özelliği taşımaktadır. Kontrolör parametrelerinin en uygun değerlerinin belirlenmesi için LCA, ACO ve OIO algoritmaları kullanılmıştır. Kullanılan 
algoritmaların sonuçlarının karşılaştırılması için çok amaçlı bir maliyet fonksiyonu kullanılmıştır.

$\mathrm{Bu}$ çalışmanın geri kalan kısmı şu şekilde düzenlenmiştir. Bölüm 2'de çalışmanın gerçekleştirildiği mikro şebeke yapısı hakkında bilgiler verilmiştir. Bölüm 3'de parametre ayarlaması için kullanılan optimizasyon yöntemleri açıklanmıştır. Bölüm 4'de kontrolörlerin performans analizi çalışmaları gerçekleştirilmiştir. Son olarak bölüm 5'de çalışmada elde edilen sonuçlar sunulmuştur.

\section{Mikro Şebeke Modeli}

Şebeke bağlantısız mikro şebeke için kontrol planlaması birçok durumda şebeke bağlantılı moda göre daha önemlidir. Bu çalışmada analiz işlemlerinin gerçekleştirildiği şebeke bağlantısız mikro şebeke sistemi şekil 1'de gösterilmiştir. Bu mikro şebeke sistem bir geleneksel dizel jeneratör (DEG), bir yakıt hücresi (FC), bir rüzgar türbin generatörü (WTG), bir batarya enerji depolama sistemi (BESS) ve bir volan enerji depolama sisteminden (FESS) oluşur. Bu sistemde mikro şebekedeki birimler sisteme güç elektroniği elemanları ile bağlanır.

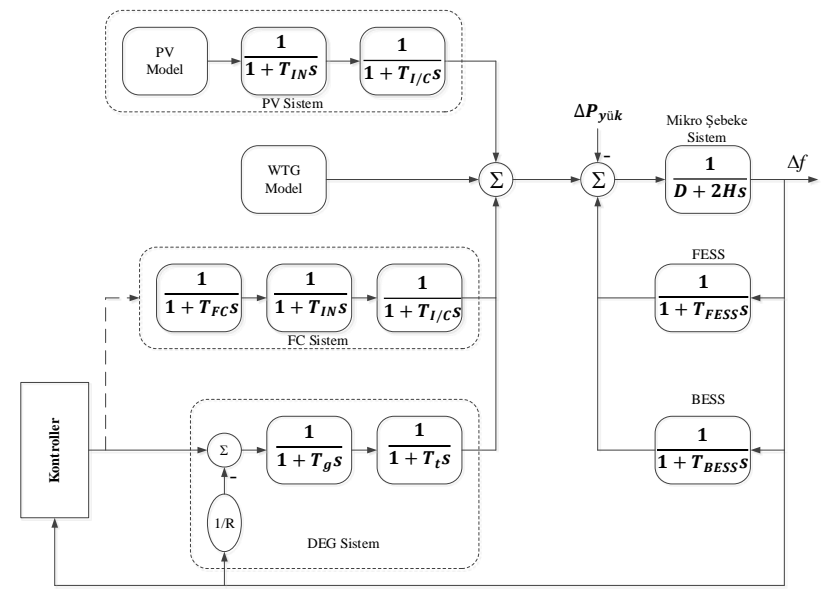

Şekil 1. Mikro şebeke için frekans yanıt modeli

Figure 1. Frequency response model for the microgrid

Pratik DEG, FC, PV, WTG, FESS ve BESS sistemlerin dinamik davranışını tam olarak simüle etmek için yüksek dereceli doğrusal olmayan matematiksel modeller kullanılmalıdır. Ancak, güç sistemi simülasyonları için genellikle transfer fonksiyonları veya basitleştirilmiş modeller kullanılır. $\mathrm{Bu}$ nedenle bu çalışmada mikro şebeke sistemi oluşturan birimler transfer fonksiyonları ile gösterilir. Örnek olarak FC sistemi 3 birimden oluşur. Birincisi yakıt hücresi, ikincisi inverter ve üçüncüsü bağlantı cihazıdır. FC sistemi oluşturan birimler geçici yanıt karekteristiklerine göre birinci derece transfer fonksiyonları ile gösterilebilirler. $\mathrm{Bu}$ nedenle, mikro şebeke frekans çalışmaları için yüksek dereceli karakteristiğe sahip FC sistemi üçüncü derece model kullanılarak gösterilebilir [22].

Şekilde 1'de gösterilen mikro şebeke sistemin birimlerine ait transfer fonksiyonları aşağıda verilir. Bu fonksiyonlara ait değerler Tablo 1'de verilmiştir.

$$
\begin{aligned}
& G_{W T G k}(s)=\frac{K_{W T G}}{1+s T_{W T G}}=\frac{\Delta P_{W T G k}}{\Delta P_{W}} \\
& G_{P V}(s)=\frac{K_{P V}}{1+s T_{P V}}=\frac{\Delta P_{P V}}{\Delta \phi} \\
& G_{F C k}(s)=\frac{K_{F C}}{1+s T_{F C}}=\frac{\Delta P_{F C k}}{\Delta P_{A E}} \\
& G_{D E G}(s)=\frac{K_{D E G}}{1+s T_{D E G}}=\frac{\Delta P_{D E G}}{\Delta f} \\
& G_{F E S S}(s)=\frac{K_{F E S S}}{1+s T_{F E S S}}=\frac{\Delta P_{F E S S}}{\Delta f} \\
& G_{B E S S}(s)=\frac{K_{B E S S}}{1+s T_{B E S S}}=\frac{\Delta P_{B E S S}}{\Delta f}
\end{aligned}
$$

Güç sisteminin kararlı çalışmasını sağlamak için toplam güç etkin bir şekilde kontrol edilmeli ve bağlı yüklerin toplam güç gereksinimleri karşılanmalıdır. Bu güç talebi referans değeri $P_{S}^{*}$ ile toplam güç üretim değeri $P_{S}$ arasındaki fark tarafindan ifade edilir.

$\Delta P_{e}=P_{s}^{*}-P_{s}$

Mikro şebeke frekansı güç değişikliğine bağlı olarak değiştiği için, sistem frekansındaki $\Delta f$ değişimi denklem 8'de verilir.

$\Delta f=\frac{\Delta P_{e}}{K_{s y s}}$

$K_{\text {sys }}$ sistem frekans1 karakteristik sabitini gösterir. Sistem frekansındaki ve güç sapmasındaki değişiklikler arasında doğal bir zaman gecikmesi olduğundan, sistem frekans1 değişimi için güç sapmasının transfer fonksiyonu, p.u. olarak denklem 9'da verilir.

$G_{s y s}(s)=\frac{\Delta f}{\Delta P_{e}}=\frac{1}{K_{s y s}\left(1+s T_{s y s}\right)}=\frac{1}{D+2 H s}$

Burada $H$ ve $D$ değerleri mikro şebeke sisteminin eşdeğer atalet sabitini ve sönümleme sabitini ifade eder $K_{W T G}, K_{P V}, K_{F C}, K_{D E G}, K_{F E S S}$ ve $K_{B E S S}$ 
değerleri analiz çalışmalarında 1 olarak alınır [4], [23].

\section{Tablo 1. Mikro şebeke sistemin parametreleri}

Table 1. The parameters of the microgrid system

\begin{tabular}{|c|c|c|}
\hline Sembol & Parametre & Değer. \\
\hline $\mathrm{D}(\mathrm{pu} / \mathrm{Hz})$ & $\begin{array}{l}\text { Mikro şebekenin } \\
\text { sönümleme sabiti }\end{array}$ & 0.015 \\
\hline $2 \mathrm{H}$ (pu.s) & $\begin{array}{l}\text { Mikro şebekenin } \\
\text { eşdeğer atalet sabiti }\end{array}$ & 0.1667 \\
\hline$T_{B E S S}(s)$ & $\begin{array}{l}\text { BESS sistemi zaman } \\
\text { sabiti }\end{array}$ & 0.1 \\
\hline$T_{F E S S}(s)$ & $\begin{array}{l}\text { FESS sistemi zaman } \\
\text { sabiti }\end{array}$ & 0.1 \\
\hline$T_{g}(s)$ & $\begin{array}{l}\text { DEG birimi governör } \\
\text { zaman sabiti }\end{array}$ & 0.08 \\
\hline$T_{t}(s)$ & $\begin{array}{l}\text { DEG birimi generatör } \\
\text { zaman sabiti }\end{array}$ & 0.4 \\
\hline$T_{F C}(s)$ & $\begin{array}{l}\text { FC birimi FC zaman } \\
\text { sabiti }\end{array}$ & 0.26 \\
\hline$T_{\bar{I}}(s)$ & $\begin{array}{l}\text { FC ve PV birimi } \\
\text { bağlantı cihazı zaman } \\
\text { sabiti }\end{array}$ & 0.004 \\
\hline$T_{I N}(s)$ & $\begin{array}{l}\text { FC ve PV birimi } \\
\text { inverter zaman sabiti }\end{array}$ & 0.04 \\
\hline$T_{P V}(s)$ & $\begin{array}{l}\text { PV birimi PV zaman } \\
\text { sabiti }\end{array}$ & 1.8 \\
\hline$T_{W T G}(s)$ & $\begin{array}{l}\text { WTG sistemi zaman } \\
\text { sabiti }\end{array}$ & 1.5 \\
\hline $\mathrm{R}(\mathrm{Hz} / \mathrm{pu})$ & $\begin{array}{l}\text { Mikro şebekenin } \\
\text { frekans düşüş faktörü }\end{array}$ & 3 \\
\hline
\end{tabular}

\section{Tamsayı Derece Yaklaşımı ile Kesir Dereceli Kontrolör}

Kesir dereceli sistemlerin rasyonel yaklaşımını yapmak amacıyla literatürde çok sayıda metot vardır. Bu metotlara Oustaloup, Carlson, Matsuda, Grünwald-Letnikoff, Maclaurin serilerine dayalı metotlar v.b. örnek gösterilebilir. CFE bu işlem için önemli metotlardan biridir ve bu çalışmada $s^{\alpha}(0<\alpha<$ 1) realizasyonunda kullanılır. CFE metodu şu şekilde gösterilebilir [24][13].

$(1+x)^{\alpha}=\frac{1}{1-} \frac{\alpha x}{1+} \frac{(1+\alpha) x}{2+} \frac{(1-\alpha) x}{3+} \frac{(2+\alpha) x}{4+} \frac{(2-\alpha) x}{5+----}(10)$

Bu formülde $s^{\alpha}$ hesabinda $x=s-1$ kullanılır. Bütün yaklaşım hesaplamaları Tablo 2'de verilir. [13].
Denklem 10 yardımı ile $\alpha$ 'ya göre 1., 2., 3. ve 4 . derece tamsayı yaklaşımı aşağıdaki gibidir.

$$
\begin{aligned}
& \frac{(1+\alpha) s+(1-\alpha)}{(1-\alpha) s+(1+\alpha)} \\
& \frac{\left(\alpha^{2}+3 \alpha+2\right) s^{2}+\left(-2 \alpha^{2}+8\right) s+\left(\alpha^{2}-3 \alpha+2\right)}{\left(\alpha^{2}-3 \alpha+2\right) s^{2}+\left(-2 \alpha^{2}+8\right) s+\left(\alpha^{2}+3 \alpha+2\right)} \\
& \left(\alpha^{3}+6 \alpha^{2}+11 \alpha+6\right) s^{3}+\left(-3 \alpha^{2}-6 \alpha^{2}+27 \alpha+54\right) s^{2} \\
& \frac{+\left(3 \alpha^{2}-6 \alpha^{2}-27 \alpha+54\right) s+\left(-\alpha^{3}+6 \alpha^{2}-11 \alpha+6\right)}{\left(-\alpha^{3}+6 \alpha^{2}-11 \alpha+6\right) s^{3}+\left(3 \alpha^{2}-6 \alpha^{2}-27 \alpha+54\right) s^{2}} \\
& \quad+\left(-3 \alpha^{2}-6 \alpha^{2}+27 \alpha+54\right) s+\left(\alpha^{3}+6 \alpha^{2}+11 \alpha+6\right) \\
& \left(\alpha^{4}+10 \alpha^{3}+35 \alpha^{2}+50 \alpha+24\right) s^{4}+\left(-4 \alpha^{4}-20 \alpha^{3}+40 \alpha^{2}+320 \alpha+384\right) s^{3} \\
& \quad+\left(6 \alpha^{4}-150 \alpha^{2}+864\right) s^{2}+\left(-4 \alpha^{4}+20 \alpha^{3}+40 \alpha^{2}-320 \alpha+384\right) s \\
& \quad+\left(\alpha^{4}-10 \alpha^{3}+35 \alpha^{2}-50 \alpha+24\right) \\
& \frac{\left(\alpha^{4}-10 \alpha^{3}+35 \alpha^{2}-50 \alpha+24\right) s^{4}+\left(-4 \alpha^{4}+20 \alpha^{3}+40 \alpha^{2}-320 \alpha+384\right) s^{3}}{\quad} \\
& \quad+\left(6 \alpha^{4}-150 \alpha^{2}+864\right) s^{2}+\left(-4 \alpha^{4}-20 \alpha^{3}+40 \alpha^{2}+320 \alpha+384\right) s
\end{aligned}
$$

$\mathrm{Bu}$ çalışmada mikro şebeke sistem için önerilen kontrolör FOPID kontrolördür. Bu kontrolör denklem $15^{\prime}$ de gösterilir. Kontrolörün $\lambda$ ve $\mu$ değerleri için gerekli tam sayı yaklaşım karşılıkları Tablo 2'de verilmiştir.

$C(s)=K_{p}+\frac{K_{i}}{s^{\lambda}}+K_{d} s^{\mu}$

\section{Optimizasyon Algoritmaları}

Gelişen sistemler ile beraber ortaya çıkan karmaşık ve çok yüksek boyutlu problemlerin çözümünde klasik optimizasyon teknikleri yetersiz kalmıştır. Bunun sonucu olarak problem çözümünde bütün çözümleri bulmak yerine olası çözümleri bulan sezgisel algoritmalar geliştirilmiştir. $\mathrm{Bu}$ algoritmalar birçok probleme uyarlanarak çözümleri basit bir şekilde bulabilirler [25]. Meta-sezgisel ve evrimsel algoritmalar tipik olarak, problemlerin büyük çoğunluğunu oluşturan ve diğer teknikler kullanılarak kolayca veya hiç çözülemeyen problemlerin yakın olan en uygum çözümlerini hesaplamak için uygulanır. Rastgele arama yapıları nedeniyle, evrimsel algoritmaların herhangi bir soruna en uygun çözümü bulması asla garanti edilmez, ancak varsa, yakın olan en uygun çözümü bulurlar. Meta-sezgisel ve evrimsel algoritmalar, çok çeşitli problemlere uygulanabilen ve problemden bağımsız tekniklerdir. "Algoritma”, bir sorunu çözmek için gerçekleştirilen bir dizi işlem anlamına gelir. Algoritmalar, belirtilen bir yakınsama ölçütüne ulaşıldığında sona eren yinelemeli işlemlerden veya adımlardan oluşur [26]. 
Tablo 2. $s^{0.1}, s^{0.2}, \ldots, s^{0.9}$ için rasyonel yaklaşım

Table 2. Rational approximations for $s^{0.1}, s^{0.2}, \ldots, s^{0.9}$

\begin{tabular}{|c|c|c|c|c|}
\hline & $\begin{array}{l}\text { 1.derece } \\
\text { yaklaşım }\end{array}$ & 2. derece yaklaşım & 3. derece yaklaşım & 4. derece yaklaşım \\
\hline \multirow[t]{2}{*}{$s^{0.1}$} & $1.22 s+1$ & $1.351 s^{2}+4.67 s+1$ & $1.444 s^{3}+11.421 s^{2}+10.333 s+1$ & $1.518 s^{4}+21.529 s^{3}+44.596 s^{2}+18.222 s+1$ \\
\hline & $\overline{s+1.22}$ & $\overline{s^{2}+4.67 s+1.351}$ & $\overline{s^{3}+10.333 s^{2}+11.421 s+1.444}$ & $\overline{s^{4}+18.222 s^{3}+44.596 s^{2}+21.529 s+1.518}$ \\
\hline \multirow[t]{2}{*}{$s^{0.2}$} & $1.5 s+1$ & $1.833 s^{2}+5.5 s+1$ & $2.1 s^{3}+14.67 s^{2}+12 s+1$ & $2.316 s^{4}+29.333 s^{3}+56 s^{2}+21 s+1$ \\
\hline & $s+1.5$ & $\overline{s^{2}+5.5 s+1.833}$ & $\overline{s^{3}+12 s^{2}+14.67 s+2.1}$ & $\overline{s^{4}+21 s^{3}+56 s^{2}+29.333 s+2.316}$ \\
\hline \multirow[t]{2}{*}{$s^{0.3}$} & $1.857 s+1$ & $2.5126 s^{2}+6.5714 s+1$ & $3.071 s^{3}+19.1344 s^{2}+14.1428 s+1$ & $3.57 s^{4}+40.63 s^{3}+71.546 s^{2}+24.57 s+1$ \\
\hline & $\overline{s+1.857}$ & $\overline{s^{2}+6.5714 s+2.5126}$ & $\overline{s^{3}+14.1428 s^{2}+19.1344 s+3.071}$ & $\overline{s^{4}+24.57 s^{3}+71.546 s^{2}+40.63 s+3.57}$ \\
\hline \multirow[t]{2}{*}{$s^{0.4}$} & $2.333 s+1$ & 3. $5 s^{2}+8 s+1$ & $4.5769 s^{3}+25.5 s^{2}+17 s+1$ & $5.594 s^{4}+57.538 s^{3}+93.5 s^{2}+29.333 s+1$ \\
\hline & $s+2.333$ & $\overline{s^{2}+8 s+3.5}$ & $\overline{s^{3}+17 s^{2}+25.5 s+4.5769}$ & $\overline{s^{4}+29.333 s^{3}+93.5 s^{2}+57.538 s+5.594}$ \\
\hline \multirow[t]{2}{*}{$s^{0.5}$} & $3 s+1$ & $5 s^{2}+10 s+1$ & $7 s^{3}+35 s^{2}+21 s+1$ & $9 s^{4}+84 s^{3}+126 s^{2}+36 s+1$ \\
\hline & $s+3$ & $\overline{s^{2}+10 s+5}$ & $\overline{s^{3}+21 s^{2}+35 s+7}$ & $s^{4}+36 s^{3}+126 s^{2}+84 s+9$ \\
\hline \multirow[t]{2}{*}{$s^{0.6}$} & $4 s+1$ & 7. $428 s^{2}+13 s+1$ & $11.143 s^{3}+50.143 s^{2}+27 s+1$ & $15.076 s^{4}+128.143 s^{3}+177.428 s^{2}+46 s+1$ \\
\hline & $\overline{s+4}$ & $\overline{s^{2}+13 s+7.428}$ & $\overline{s^{3}+27 s^{2}+50.143 s+11.143}$ & $\overline{s^{4}+46 s^{3}+177.428 s^{2}+128.143 s+15.076}$ \\
\hline \multirow[t]{2}{*}{$s^{0.7}$} & $5.67 s+1$ & $11.77 s^{2}+18 s+1$ & $18.933 s^{3}+76.846 s^{2}+37 s+1$ & $26.965 s^{4}+209.38 s^{3}+267.54 s^{2}+62.67 s+1$ \\
\hline & $s+5.67$ & $\overline{s^{2}+18 s+11.77}$ & $\overline{s^{3}+37 s^{2}+76.846 s+18.933}$ & $\overline{s^{4}+62.67 s^{3}+267.54 s^{2}+209.38 s+26.965}$ \\
\hline \multirow[t]{2}{*}{$s^{0.8}$} & $9 s+1$ & $21 s^{2}+28 s+1$ & $36.27 s^{3}+133 s^{2}+57 s+1$ & $54.41 s^{4}+386.91 s^{3}+456 s^{2}+96 s+1$ \\
\hline & $s+9$ & $\overline{s^{2}+28 s+21}$ & $\overline{s^{3}+57 s^{2}+133 s+36.27}$ & $s^{4}+96 s^{3}+456 s^{2}+386.91 s+54.41$ \\
\hline \multirow[t]{2}{*}{$s^{0.9}$} & $19 s+1$ & 50. $1 s^{2}+58 s+1$ & $93.026 s^{3}+308.45 s^{2}+117 s+1$ & $147.04 s^{4}+959.64 s^{3}+1042.36 s^{2}+196 s+1$ \\
\hline & $s+19$ & $\overline{s^{2}+58 s+50.1}$ & $\overline{s^{3}+117 s^{2}+308.45 s+93.026}$ & $\overline{s^{4}+196 s^{3}+1042.36 s^{2}+959.64 s+147.04}$ \\
\hline
\end{tabular}

\section{Lig Şampiyonası Algoritması}

LCA spor tabanlı bir metasezgisel algoritmadir [27]. LCA spor liglerindeki şampiyonluk sürecini taklit eden bir algoritmadır. Spor takımları olarak rol oynayan bireyler, yapay bir ligde birkaç hafta boyunca rekabet ederler (iterasyon). Her hafta lig programına bağlı olarak, takımlar çift olarak oynar ve oyun sonucu kazanma, kaybetme ve beraberlik açısından belirlenir, her takım tarafindan takip edilen belirli takım formasyonu/düzenlemesi (çözüm) ile birlikte oyun gücü verilir. Önceki hafta etkinliklerini takip eden her takım, bir sonraki hafta yarışması için formasyon/oyun tarzında (yeni bir çözüm üretilir) gerekli değişiklikleri yapar ve şampiyona birkaç sezon boyunca devam eder (durma koşulu). LCA popülasyona dayalı bir algoritmadır ve burada takımlar parçacık sürü optimizasyonunun (PSO) "parçacıklarına" benzerdir ancak aramalarını gerçekleştirmenin oldukça farklı bir yoluna sahiptirler. Bir LCA'nın takımıyla ilişkili yeni bir çözümün üretilme şekli, yaklaşan maçları için uygun bir düzenleme tasarlayarak koçlar tarafindan takip edilen maç analizi sürecini taklit ederek yönetilir. Tipik bir maç analizinde, koçlar kendi oyun deneyimleri ve rakiplerinin oyun tarzı temelinde düzenlemelerini değiştireceklerdir [25], [27], [28].

\section{Karınca Kolonisi Algoritması}

ACO meta sezgisel bir algoritma olup popülasyon temelli bir yaklaşımdır. Bu algoritma Dorigo ve arkadaşları tarafından ayrık optimizasyon problemlerini çözmek için kullanılmıştır [29]. ACO gerçek karıncaların bir besin kaynağı ile yuvaları arasındaki en kısa yolu bulma şeklini taklit eder. Karıncalar birbirleriyle feromon izleri aracılığıyla iletişim kurar ve hangi yolun izleneceği konusunda bilgi alışverişinde bulunurlar. Karınca sayısı belirli bir yolu ne kadar çok izlerse, bu yol (iz) o kadar çekici hale gelir ve bunu kendi feromonlarını bırakarak diğer karıncalar izler.

\section{Optikten Esinlenen Optimizasyon}

OIO algoritması içbükey ve dışbükey aynaların optik özelliklerine dayanan fiziksel tabanlı bir optimizasyon algoritmasıdır. Fizikte içbükey yüzeylerin/aynaların 1şık 1şınlarını ana eksene doğru yansıttığı gözlemlenmiştir (aynanın ortasından geçen ve aynaya dik bir çizgi). Böyle bir ayna ışı 1 ışınlarının birleşmesine neden olur. 
Diğer taraftan, dışbükey bir yüzey/ayna ile ana eksene paralel gelen 1şı uzağa yansıtılır. Böylece 1şık 1şınlarının hepsi ayrıșıyor gibi görünür. Bu fiziksel kurallar ile, OIO algoritması sayısal optimizasyonun arama sürecini gerçekleştirir. OIO, optimize edilecek sayısal işlevin yüzeyini yansıtıcı bir yüzey olarak ele alır; burada her tepe noktası dışbükey bir ayna olarak yansitılır ve her vadi içbükey bir ayna olarak yansıtılır. Arama alanı içinde bir çözüm $\left(R^{n}\right.$ 'de bir alt küme) olarak eşlenen ortak arama/çözüm ve hedef alandaki $\left(R^{n+1}\right.$ 'de bir alt küme) her noktanın yapay bir sşık noktası olduğu varsayılır. Bu șekilde, yapay bir 1ş1k noktasından parıldayan yapay 1șın, yansitıcı yüzey kısmen bir zirvenin bir parçası veya bir vadinin bir parçası olması ve yapay görüntü noktasına (arama/çözüm uzayında yeni bir çözüm olarak eşlenen amaç uzayında ve ortak aramada yeni bir nokta ) dik olarak (arama alanındaki 1şık noktası konumuna doğru) veya ters olarak (arama alanındaki 1şık noktası konumun dişına doğru) oluşturulması ile verilen işlev yüzeyi tarafindan yapay olarak geri yansitılır. Böyle bir model, optimum arayış sırasında hem keşif hem de keşif görevlerini yerine getirebilmemizi sağlar [30], [31].

\section{Performans Kriteri}

Kontrolörlerin parametre değerleri optimizasyon algoritmaları tarafindan optimizasyon probleminin çözümü için bulunur. Bu nedenle en uygun optimizasyon çözümünün elde edilmesi için bir fonksiyon tanımlanmalıdır ve bu fonksiyona amaç fonksiyonu adı verilir. PID parametrelerinin belirlenmesinde kullanilan algoritmaların optimizasyonu için literatürde hata karelerinin toplamı (Integral Squared Error (ISE)), mutlak hatanın toplamı (Integral Absolute Error (IAE)), zaman ağırlıklı mutlak hatanın toplamı (Integral Time-weighted Absolute Error (ITAE)), zaman ağırlıklı hata karelerinin toplamı (Integral Time-weighted Squared Error (ITSE)), gibi fonksiyonlar önerilmiştir [32]. $\mathrm{Bu}$ fonksiyonların denklemleri aşağıda verilmiştir.

$$
\begin{aligned}
& I A E=\int_{0}^{\infty}|e(t)| d t \\
& I T A E=\int_{0}^{\infty} t|e(t)| d t \\
& I T S E=\int_{0}^{\infty} t e^{2}(t) d t \\
& I S E=\int_{0}^{\infty} e^{2}(t) d t
\end{aligned}
$$

$\mathrm{Bu}$ çalıșmada çok amaçlı bir performans kriteri kontrol kazançlarını bulmak için kullanılır. $\mathrm{Bu}$ kriter yerleşme zamanı $t_{s}$, maksimum aşma $M_{P}$, sürekli durum hatası $E_{S S}$, ITAE ve sönümleme oranı $\zeta$ değerlerinden meydana gelir. $\mathrm{Bu}$ çalışmada kullanılan performans kriteri $J(k)$ denklem 17 de verilir.

$$
\begin{aligned}
& J(k)=w_{1} M_{P}+w_{2} t_{s}+w_{3} E_{S S}+w_{4} I T S E+ \\
& w_{5} / \zeta
\end{aligned}
$$

Bu performans kriteri (17) 6 terimden oluşur ve her birinin önemi bir ağırlık faktörü $w_{i}$ tarafından belirlenir. $\mathrm{Bu}$ ağırlık faktörlerinin değerleri istenilen özelliklere uygun olarak bu çalışmada $w_{1}=1, \quad w_{2}=1, \quad w_{3}=1000, \quad w_{4}=500$ ve $w_{5}=0.25$ olarak seçilmiştir. Seçilen $w_{i}$ değerlerindeki bir artış bu değer ile ilişkili özellikte bir gelişme ile sonuçlanacaktır. Ancak bu özellikteki gelişme diğer özelliklerde bir azalmaya yol açabilir [33].

\section{Benzetim Sonuçları}

Mikro şebeke sistem önerilen çok amaçlı maliyet fonksiyonu kullanılarak 3 farklı optimizasyon algoritması ile ayrı ayrı optimize edilmiştir. Optimizasyon işlemleri sonrası elde edilen kontrolör parametre değerleri Tablo 3'de verilmiştir. Çok amaçlı maliyet fonksiyonunu oluşturmak için kullanılan verilere ait değerler ve maliyet fonksiyonu sonuçları Tablo 4'de verilmiștir.

Tablo 4 verileri incelendiği zaman en iyi maliyet değeri ve sürekli durum hatası LCA için elde edilirken en kötü sonuç ACO için elde edilmiştir. ACO ile en iyi maksimum aşma değeri elde edilmiştir. Maksimum aşma hariç bütün değerler için en iyi sonuçlar LCA ile elde edilmiştir. OIO ile maksimum aşma için en kötü değer elde edilmiştir. En kötü sönümleme oranı, sürekli duruma erişme süresi ve sürekli durum hatası değeri ACO için elde edilmiștir.

Şekil 5'de farklı optimizasyon yöntemleri ile parametre değerleri optimize edilen IOA FOPID kontrolör için mikro şebekenin frekansının zamana göre değişimi gösterilmiştir. Şekil incelendiği zaman elde edilen sonuçların Tablo 4 değerleri ile uygun olduğu görülmektedir. Tablo 4'e göre en kötü sönümleme oranına sahip ACO algoritması için sistemin zaman yanıtı daha fazla salınımlara sahiptir. Şekilde yine en fazla aşma değerinin Tablo değerlerine uygun olarak OIO için elde edildiği görülmektedir. Şekilden görüleceği üzere kontrolör 
LCA algoritması ile elde edilen değerlere sahip iken . en uygun dinamik zaman yanıtına sahiptir.

Tablo 3. IOA FODPID kontrollerin hesaplanan parametre değerleri

Table 3. Calculated parameter values of IOA FOPID controller

\begin{tabular}{cccc}
\hline Kontroller & LSA & KKA & OEO \\
\hline FC kontroller $\boldsymbol{K}_{\boldsymbol{p}}$ & 4.8518 & 2.5253 & 0.2495 \\
FC kontroller $\boldsymbol{K}_{\boldsymbol{i}}$ & 9.5746 & 6.9697 & 7.6632 \\
FC kontroller $\boldsymbol{\lambda}$ & 0.9 & 0.9 & 0.9 \\
FC kontroller $\boldsymbol{\lambda} \boldsymbol{d e r} \boldsymbol{e c} \boldsymbol{e}$ & 1 & 2 & 1 \\
FC kontroller $\boldsymbol{K}_{\boldsymbol{d}}$ & 0.8596 & 1.6162 & 1.4832 \\
FC kontroller $\boldsymbol{\mu}$ & 0.9 & 0.8 & 0.4 \\
FC kontroller $\boldsymbol{\mu}$ derece & 1 & 1 & 1 \\
DEG kontroller $\boldsymbol{K}_{\boldsymbol{p}}$ & 0.0094 & 8.2828 & 0.3985 \\
DEG kontroller $\boldsymbol{K}_{\boldsymbol{i}}$ & 9.9815 & 9.8989 & 6.9804 \\
DEG kontroller $\boldsymbol{\lambda}$ & 0.9 & 0.9 & 0.8 \\
DEG kontroller $\boldsymbol{\lambda} \boldsymbol{d e r} \boldsymbol{e c} \boldsymbol{e}$ & 1 & 3 & 1 \\
DEG kontroller $\boldsymbol{K}_{\boldsymbol{d}}$ & 0.0426 & 2.3233 & 0.3324 \\
DEG kontroller $\boldsymbol{\mu}$ & 0.1 & 0.9 & 0.3 \\
DEG kontroller $\boldsymbol{\mu}$ derece & 2 & 1 & 2
\end{tabular}

Tablo 4. Farklı optimizasyon algoritmaları için elde edilen değerlerin karşılaştırılması

Table 4. Comparison of the obtained values for different optimization algorithms

\begin{tabular}{ccccccc}
\hline $\begin{array}{c}\text { Optimizasyon } \\
\text { yöntemi }\end{array}$ & $\boldsymbol{M}_{\boldsymbol{p}}$ & $\boldsymbol{t}_{\boldsymbol{s}}$ & $\boldsymbol{E}_{\boldsymbol{S S}}$ & ITSE & $\boldsymbol{\zeta}$ & $\boldsymbol{J}(\boldsymbol{k})$ \\
\hline LCA & 0.034 & 0.5424 & $3.549 \mathrm{e}-05$ & $1.538 \mathrm{e}-05$ & 0.3052 & 1.4387 \\
ACO & 0.0248 & 2.0006 & $8.5799 \mathrm{e}-05$ & $1.7864 \mathrm{e}-05$ & 0.1115 & 4.3623 \\
OIO & 0.048 & 1.5487 & $7.493 \mathrm{e}-05$ & $6.586 \mathrm{e}-05$ & 0.1934 & 2.9972
\end{tabular}

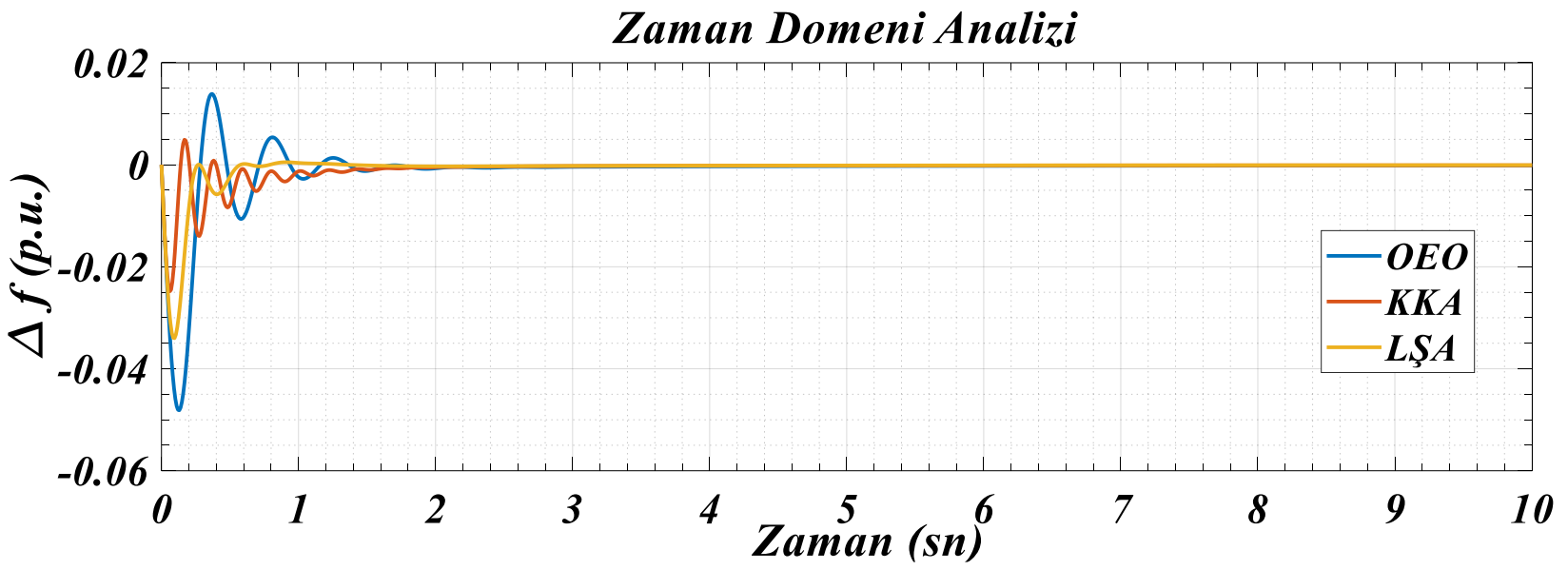

Şekil 3. Farklı optimizasyon algoritmaları ile tasarlanan kontrolör için mikro şebekenin zaman yanitı

Figure 3. Time response of the microgrid for the controller designed with different optimization algorithms 


\section{Sonuçlar}

Bu çalışmada, mikro şebeke yük frekans kontrolü için CFE metodu kullanılarak elde edilen bir IOA FODPID kontrolörün uygulaması gösterilmiştir. Önerilen IOA FOPID kontrolörün en uygun parametre değerlerinin belirlenmesi için LCA, ACO ve OIO algoritmaları kullanılmıştır. Farklı optimizasyon algoritmaları ile en uygun parametre değerlerinin belirlenmesi için çok amaçlı bir maliyet fonksiyonu tanımlanmıştır. Çalışmada elde edilen sonuçlara göre IOA FOPID kontrolörün LCA ile optimizasyonu ile en iyi çok amaçlı maliyet fonksiyonu değeri elde edilmiştir. Ayrıca çok amaçlı maliyet fonksiyonunu olușturan değerlerden sürekli duruma erişme süresi, sürekli durum hatası, sönümleme oranı ve ITSE maliyet değeri için en iyi sonuçlar LCA algoritması ile elde edilmiştir. Bütün optimizasyon algoritmaları için gerçekleştirilen zaman domeni cevaplarından görüleceği üzere IOA FOPID kontrolör mikro şebeke LFC uygulamalarında başarılı bir şekilde kullanılabilir.

\section{Kaynaklar}

[1] S. Chowdhury, S. P. Chowdhury, and P. Crossley, Microgrids and active distribution networks. The Institution of Engineering and Technology, 2009.

[2] R. H. Lasseter et al., "CERTS microgrid laboratory test bed," IEEE Trans. Power Deliv., vol. 26, no. 1, pp. 325-332, 2011.

[3] R. Lasseter et al., "The CERTS MicroGrid Concept," California, 2002.

[4] H. Bevrani, F. Habibi, P. Babahajyani, M. Watanabe, and Y. Mitani, "Intelligent frequency control in an AC microgrid: Online PSO-based fuzzy tuning approach," IEEE Trans. Smart Grid, vol. 3, no. 4, pp. 1935-1944, 2012.

[5] B. Yıldırım and M. T. Gençoğlu, "Oscillatory stability and eigenvalue analysis of power system with microgrid," Electr. Eng., 2018.

[6] M. Mordjaoui, S. Haddad, A. Medoued, and A. Laouafi, "Electric load forecasting by using dynamic neural network," Int. J. Hydrogen Energy, vol. 42, no. 28, pp. 17655-17663, Jul. 2017.

[7] S. Elkawafi, A. Khalil, A. I. Elgaiyar, and J. Wang, "Delay-dependent stability of LFC in Microgrid with varying time delays," in 2016 22nd International Conference on Automation and Computing (ICAC), 2016, pp. 354-359.
P. C. Sahu, S. Mishra, R. C. Prusty, and S. Panda, "Improved-salp swarm optimized type-II fuzzy controller in load frequency control of multi area islanded AC microgrid," Sustain. Energy, Grids Networks, vol. 16, pp. 380-392, Dec. 2018.

[9] A. Rezvani, M. Izadbakhsh, and M. Gandomkar, "Microgrid dynamic responses enhancement using artificial neural network-genetic algorithm for photovoltaic system and fuzzy controller for high wind speeds," Int. J. Numer. Model.

Electron. Networks, Devices Fields, vol. 29, no. 2, pp. 309-332, Mar. 2016.

[10] X. Zhang, D. Wang, T. Yu, Z. Xu, and Z. Fan, "Ensemble learning for optimal active power control of distributed energy resources and thermostatically controlled loads in an islanded microgrid," Int. J. Hydrogen Energy, vol. 43, no. 49, pp. 22474-22486, Dec. 2018.

[11] T. Vigneysh and N. Kumarappan, "Autonomous operation and control of photovoltaic/solid oxide fuel cell/battery energy storage based microgrid using fuzzy logic controller," Int. J. Hydrogen Energy, vol. 41, no. 3, pp. 1877-1891, Jan. 2016.

F. Banis, D. Guericke, H. Madsen, and N. K. Poulsen, "Load-frequency control in microgrids using target-adjusted MPC," IET Renew. Power Gener., vol. 14, no. 1, pp. 118-124, Jan. 2020.

M. M. Özyetkin and N. Tan, "Integer order approximation of fractional order systems," in Signal Processing and Communications Applications Conference (SIU) IEEE, 2010, pp. 949-952.

[14] A. Yüce, F. N. Deniz, and N. Tan, "A New Integer Order Approximation Table for Fractional Order Derivative Operators," IFACPapersOnLine, 2017.

[15] B. M. Vinagre, I. Podlubny, A. Hernandez, and V. Feliu, "Some Approximations of Fractional Order Operators Used in Control Theory and Applications," Fract. Calc. Appl. Anal., 2000.

[16] A. Oustaloup, F. Levron, B. Mathieu, and F. M. Nanot, "Frequency-band complex noninteger differentiator: Characterization and synthesis," IEEE Trans. Circuits Syst. I Fundam. Theory Appl., 2000.

[17] K. Matsuda and H. Fujii, "H(infinity) optimized wave-absorbing control - Analytical and experimental results," J. Guid. Control. Dyn., 2008.

[18] B. T. Krishna, "Studies on fractional order differentiators and integrators: A survey," Signal Processing, 2011.

[19] Y. Q. Chen, I. Petráš, and D. Xue, "Fractional order control - A tutorial," in Proceedings of the American Control Conference, 2009. 
[20] G. E. Carlson and C. A. Halijak, "Approximation of Fractional Capacitors (1/s)1/n by a Regular Newton Process," IEEE Trans. Circuit Theory, 1964.

[21] B. YILDIRIM, M. T. OZDEMIR, and I. EKE, "Design of Integer Order Approximation Fractional Order Controller with for Automatic Voltage Regulation System," in 2019 4th International Conference on Power Electronics and their Applications (ICPEA), 2019, pp. 1-6.

[22] S. Obara, "Analysis of a fuel cell micro-grid with a small-scale wind turbine generator," Int. J. Hydrogen Energy, vol. 32, no. 3, pp. 323-336, 2007.

[23] D. J. Lee and L. Wang, "Small-signal stability analysis of an autonomous hybrid renewable energy power generation/energy storage system part I: Time-domain simulations," IEEE Trans. Energy Convers., vol. 23, no. 1, pp. 311-320, 2008.

[24] B. T. Krishna and K. V. V. S. Reddy, "Active and passive realization of fractance device of order 1/2," Act. Passiv. Electron. Components, 2008.

[25] M. T. Özdemir, B. Yıldırım, H. Gülan, and M. T. Gençoğlu, "Automatic generation control in an AC isolated microgrid using the league championship," Sci. Eng. J Firat Univ., vol. 29, pp. 109-120, 2017.

[26] O. Bozorg-Haddad, M. Solgi, and H. A. Loáiciga, Meta-Heuristic and Evolutionary Algorithms for Engineering Optimization. 2017.
[27] A. Husseinzadeh Kashan, "League Championship Algorithm (LCA): An algorithm for global optimization inspired by sport championships," Appl. Soft Comput., vol. 16, pp. 171-200, Mar. 2014.

[28] H. Bingöl and B. Alataş, "Metasezgisel Optimizasyon Tekniklerine Spor Tabanlı Yeni Bir Yaklaşım: Lig Şampiyonası Algoritması," Fırat Ünv. Fen Bilim. Derg., vol. 27, no. 1, pp. 1-11, 2015.

[29] M. Dorigo and L. M. Gambardella, “Ant colony system: a cooperative learning approach to the traveling salesman problem," IEEE Trans. Evol. Comput., vol. 1, no. 1, pp. 53-66, 1997.

[30] A. H. Kashan, "Computers \& Operations Research A new metaheuristic for optimization : Optics inspired optimization ( OIO )," vol. 55, pp. 99-125, 2015.

[31] M. T. Özdemir and D. Öztürk, “Comparative performance analysis of optimal PID parameters tuning based on the optics inspired optimization methods for automatic generation control," Energies, vol. 10, no. 12, 2017.

[32] E. Kılıç and M. T. Özdemir, "Güç Sistemlerindeki Optimum Otomatik Gerilim Regülasyonu için Çoklu Amaç Fonksiyonunun Belirlenmesi,” DÜMF Mühendislik Derg., 2019.

[33] M. Zamani, M. Karimi-Ghartemani, N. Sadati, and M. Parniani, "Design of a fractional order PID controller for an AVR using particle swarm optimization," Control Eng. Pract., 2009. 